\title{
LIKE-SIGN DILEPTON SEARCH FOR CHARGINO-NEUTRALINO PRODUCTION AT CDF
}

\author{
MATTHEW WORCESTER, JANE NACHTMAN, DAVID SALTZBERG \\ Department of Physics and Astronomy, University of California, Los Angeles \\ 405 Hilgard Avenue, Los Angeles, CA 90095-1547, USA \\ for the CDF Collaboration
}

\begin{abstract}
We present new results from the like-sign dilepton search for chargino-neutralino $\left(\tilde{\chi}_{1}^{ \pm} \tilde{\chi}_{2}^{0}\right)$ production in $\mathrm{p} \overline{\mathrm{p}}$ collisions at $\sqrt{s}=1.8 \mathrm{TeV}$, which complements the previously published trilepton search with the CDF detector using Fermilab Run IB data. Monte Carlo calculations of signal efficiency and backgrounds, as well as estimates of certain backgrounds taken from Run IB data, indicate a significant increase in sensitivity to $\tilde{\chi}_{1}^{ \pm} \tilde{\chi}_{2}^{0}$ production compared to the traditional trilepton analysis alone.
\end{abstract}

\section{Introduction}

Previous searches for $\tilde{\chi}_{1}^{ \pm} \tilde{\chi}_{2}^{0}$ production at the Tevatron have focused primarily on signatures with three charged leptons (trileptons) plus missing transverse energy $\left(\mathbb{E}_{T}\right) .^{1}$ In the Minimal Supersymmetric (SUSY) Standard Model, $\tilde{\chi}_{1}^{ \pm} \tilde{\chi}_{2}^{0}$ production occurs in proton-antiproton $(\mathrm{p} \overline{\mathrm{p}})$ collisions via a virtual $\mathrm{W}$ (s-channel) or a virtual squark (t-channel). In a representative minimal Supergravity model (parameters: $\mu<0, \tan \beta=2, A_{0}=0, m_{0}=200 \mathrm{GeV} / c^{2}, m_{1 / 2}=90-140 \mathrm{GeV} / c^{2}$ ), we expect three-body chargino and neutralino decays through virtual bosons and sleptons in a chargino mass region of $80-130 \mathrm{GeV} / c^{2}$. For conserved R-parity, these decays produce a distinct signature: trileptons plus $\mathbb{E}_{T}$ from a neutrino and the lightest supersymmetric particle. We demonstrate that the sensitivity to this signature can be significantly increased by searching for events with two like-sign leptons. The like-sign dilepton (LSD) search provides a strong rejection of Standard Model background through the like-sign requirement, and enhances the acceptance of the signal by requiring only two of the three leptons produced in the $\tilde{\chi}_{1}^{ \pm} \tilde{\chi}_{2}^{0}$ decay.

\section{Like-Sign Dilepton Analysis}

The LSD analysis is described in detail elsewhere. ${ }^{2}$ Here we focus on estimating the important LSD background from "fake" leptons. 

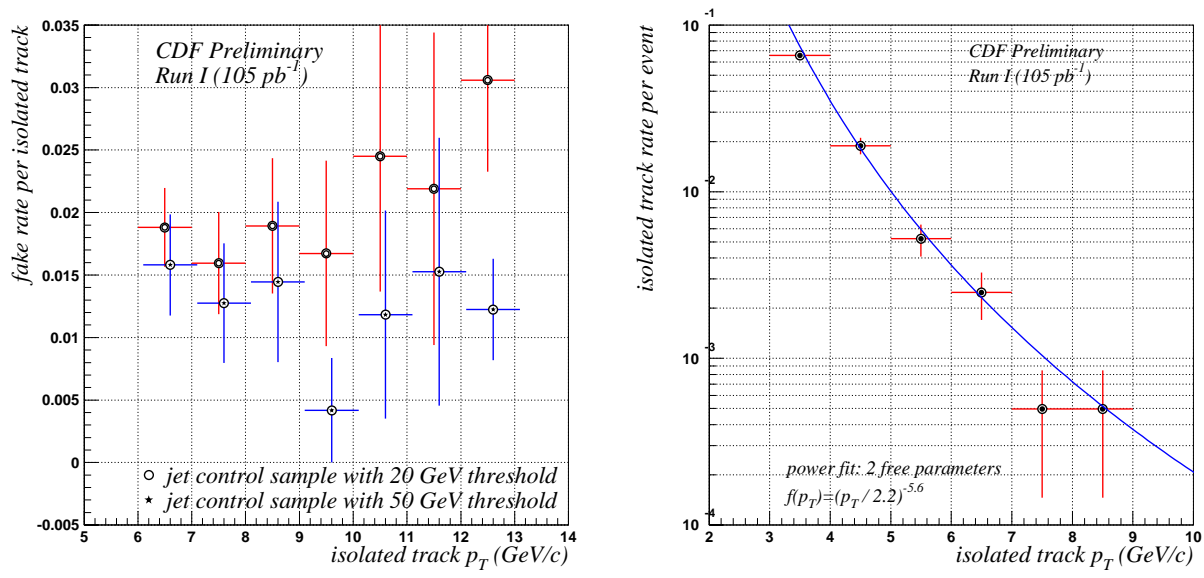

Fig. 1. Left: Fake rate per isolated track as a function of isolated track $p_{T}$. Right: Isolated track rate per event as a function of isolated track $p_{T}$. Errors shown are statistical.

The largest source of non-SUSY background estimated from data is events with one true lepton, such as $\mathrm{W} \rightarrow \ell \nu+$ jets, and a "fake" lepton, i.e. an isolated track misidentified as a lepton. This fake lepton, in combination with the true lepton from the $\mathrm{W}$ decay, can be selected as a signal event in this analysis. To estimate this background, we create both minimum bias and jet control samples with 20 and $50 \mathrm{GeV}$ thresholds with $\mathrm{W} / \mathrm{Z}$ contamination removed, and select tracks which pass our lepton ID requirements. The tracks which pass the lepton ID cuts yield the probability of an isolated track to be misidentified as a lepton, or "fake rate" per isolated track: $1.5 \%$, independent of track $p_{T}$, as shown in Figure 1. The uncertainty of this "fake rate" probability is a combination of statistical (due to few events in our samples at high $p_{T}$ ) and systematic. The systematic error is taken to account for the possible difference between the 20 and $50 \mathrm{GeV}$ jet samples, which are not expected to be identical, although both samples are consistent with $1.5 \%$ per track.

We next look at the underlying isolated tracks in $\mathrm{Z} \rightarrow \ell^{+} \ell^{-}$events, which we assume provide a model for $\mathrm{W} \rightarrow \ell \nu$ events. The isolated track rate per event is shown in Figure 1 as a function of isolated track $p_{T}$. We multiply the fake probability of $1.5 \%$ by the isolated track rate found in the $\mathrm{Z} \rightarrow \ell^{+} \ell^{-}$events, by the number of $\mathrm{W} \rightarrow \ell \nu+$ jets events expected, ${ }^{3}$ and by a factor of 0.5 for the like-sign requirement. This number of expected "fake" events drops rapidly with an increasing minimum $p_{T}$ requirement, as shown in Figure 2. Optimization of the number of expected background events as a function of the $p_{T}$ requirement yields $0.24 \pm 0.21$ events expected above $p_{T}>11 \mathrm{GeV} / c$ from $\mathrm{W} \rightarrow \ell \nu$ events in $105 \mathrm{pb}^{-1}$ of data. 


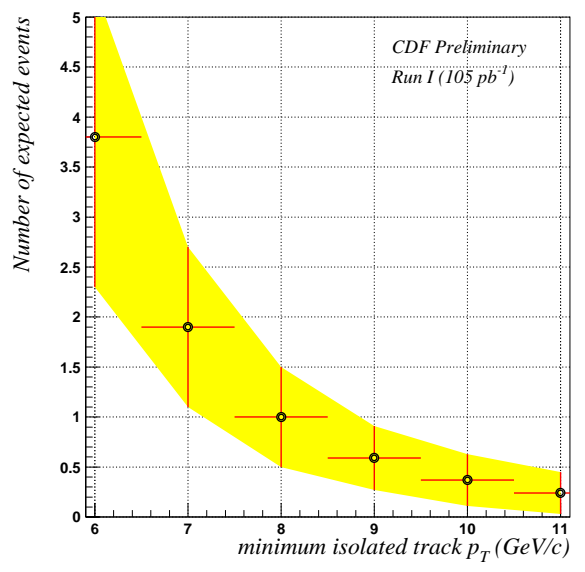

Fig. 2. Number of expected events from fakes as a function of minimum $p_{T}$ cut. Errors shown are statistical and systematic.

\section{Results}

Applying the analysis requirements and normalizing the luminosity to $105 \mathrm{pb}^{-1}$, the total expected LSD background is $0.50_{-0.32}^{+0.42}$ events, as shown in Table 1. There is little background overlap of the trilepton and LSD analyses in the selected events based on Monte Carlo studies, so they add independently. For the trilepton analysis, the expected background for the same luminosity is $1.2 \pm 0.2$ events. $^{1}$ The total expected background for the combined LSD and trilepton analyses is $1.7_{-0.5}^{+0.6}$ events.

Table 1. Background estimates for the number of events expected in $105 \mathrm{pb}^{-1}$ of data based on Monte Carlo (except for $\mathrm{W}+$ jets estimation). The MC errors are one-sigma statistical errors.

\begin{tabular}{ccl}
\hline Process & Luminosity $\left(\mathrm{pb}^{-1}\right)$ & Expected events \\
\hline $\mathrm{WZ}$ & 16,684 & $0.11 \pm 0.02$ \\
$\mathrm{ZZ}$ & 13,992 & $0.01 \pm 0.01$ \\
$\mathrm{WW}$ & 6,870 & $0_{-0}^{+0.02}$ \\
$\mathrm{t} \overline{\mathrm{t}}$ & 5,558 & $0_{-0}^{+0.02}$ \\
Drell-Yan $\left(\gamma^{*} / \mathrm{Z}\right)$ & 1,728 & $0.11_{-0.06}^{+0.10}$ \\
$\mathrm{~b} \overline{\mathrm{b}}, \mathrm{c} \overline{\mathrm{c}}$ & 3,122 & $0.03_{-0.02}^{+0.04}$ \\
$\mathrm{~W}+$ jets & (from data) & $0.24 \pm 0.21$ \\
\hline Total & & $0.50_{-0.32}^{+0.42}$ \\
\hline
\end{tabular}

Figure 3 shows the average expected limit normalized to $100 \mathrm{pb}^{-1}$ for the trilepton, LSD, and combined analyses. These limits were calculated from the signal efficiencies given in Ref. 2 and from the expected number of background events in Table 1, taking into account the signal overlap between the trilepton and LSD analyses. 

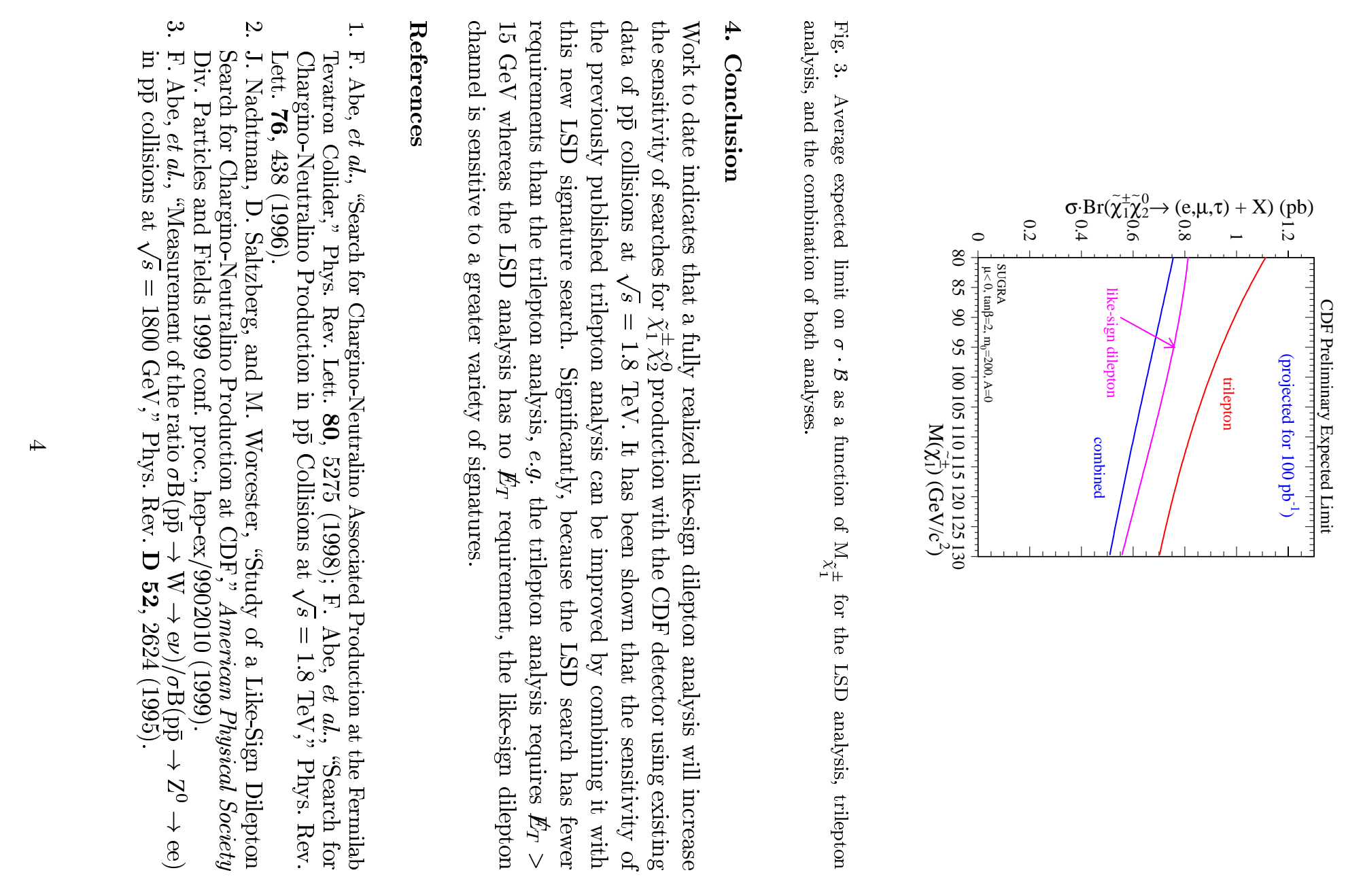